\title{
THE PROGNOSTIC VALUE OF THE SERUM INFLAMMATORY BIOMARKERS IN PATIENTS WITH CAROTID ATHEROSCLEROSIS
}

\author{
Olga Dubenko $\bowtie$ \\ Department of Neurology and Neurosurgery ${ }^{l}$ \\ olgadubenko05@gmail.com \\ Tetyana Litovchenko \\ Department of Neurology and child Neurology ${ }^{1}$ \\ Victoria Anysienkova \\ Department of Neurology and Neurosurgery ${ }^{l}$ \\ Maryna Nessonova \\ Department of Educational and Information Technologies \\ National University of Pharmacy \\ 53 Pushkinska str., Kharkiv, Ukraine, 61002 \\ Liudmyla Kovalenko \\ Department of General Practice - Family Medicine \\ V. N. Karazin Kharkiv National University \\ 4 Svobody sq., Kharkiv, Ukraine, 61022 \\ ${ }^{1}$ Kharkiv Medical Academy of Postgraduate Education \\ 58 Amosova str., Kharkiv, Ukraine, 61176
}

$\triangle$ Corresponding author

Abstract

$20 \%$ of ischemic stroke appear to originate from carotid artery atherosclerotic disease. Serum biomarkers reflecting the activity of atherosclerotic process and may help for estimate risk of acute cerebrovascular events. Several serum inflammatory markers have been proposed for risk assessment, but their prognostic role less known.

The aim of this study is to clarify the prognostic value of biomarkers of atherosclerosis lipoprotein-associated phospholipase A2 (Lp-PLA2) and E-selectin in patients with symptomatic and asymptomatic carotid stenosis.

Materials and methods. The study involved 106 patients with atherosclerotic carotid stenosis $>50 \%$ (74 men and 32 women, mean age $62.6 \pm 0.9$ ) from which 76 symptomatic ( 35 with acute ipsilateral atherothrombotic stroke and 41 after carotid endarterectomy) and 30 asymptomatic patients. The control group consisted of age- and sex-matched 20 healthy subjects. The level of serum Lp-PLA2 and E-selectin was determined using a commercially available enzyme-linked immunosorbent assay kit.

Results. The level of Lp-PLA 2 was in general significantly higher $(p<0.05)$ in patients groups than in the control group and most high Lp-PLA 2 concentration was in groups of symptomatic patients who underwent carotid endarterectomy. The level of E-selectin in the study patients was significantly higher than in the control group $(p<0.05)$. The correlation of Lp-PLA 2 with E-selectin was significant for total patients $(R=0.365664, p=0.00085)$ and group after carotid endarterectomy $(R=0.429143$, $p=0.01796)$, but not for asymptomatic group $(p>0.05)$. Receiver Operating Characteristics curves of logistic regression models which takes into joint both indicators was specificity and sensitive for predicting the occurrence of ischemic stroke.

Conclusion. Conducted study show that the levels of Lp-PLA 2 and E-selectin have a significant impact on the development of stroke in patients with atherosclerotic carotid stenosis and can be used to predict it. A multidimensional model of the dependence of the probability of stroke on a linear combination of Lp-PLA 2 and E-selectin allows to obtaining significantly higher characteristics of the accuracy of stroke prediction than models with each factor alone.

Keywords: atherosclerotic carotid stenosis, atherosclerotic markers, lipoprotein-associated phospholipase A2, E-selectin, risk of stroke.

DOI: $10.21303 / 2504-5679.2021 .001969$

\section{Introduction}

Carotid artery atherosclerotic stenosis is a common cause of ischemic stroke [1]. Around $20 \%$ of it appear to originate from carotid plaques. It is well recognized that the presence of 
atherosclerotic disease in the carotid arteries increases a substantial risk of ipsilateral cerebrovascular events, with reported annual ischemic stroke rates ranging from $0.35 \%$ to $1.3 \%$ in asymptomatic patients with moderate stenosis to approximately $5 \%$ for severe asymptomatic carotid artery stenosis [2]. Clinical, biochemical, and ultrasound markers, magnetic resonance (MRI) plaque characteristics or transcranial cerebral Doppler signals have been proposed as indicators of a high-risk plaque. It is well accepted that circulating biomarkers, including C-reactive protein and interleukin-6, reliably predict major cardiovascular events, including myocardial infarction or death. However, the relevance of biomarkers of systemic inflammation to atherosclerosis progression in the carotid artery is less established. Other inflammatory biomarkers, lipids, interleukins, homocysteine, and adipokines may be useful in quantifying carotid disease-related risk. Some serum biomarkers combined with image features may assist vascular specialists in selecting patients at high risk for stroke [3-5].

The aim of this study to clarify the prognostic value of biomarkers of atherosclerosis lipoprotein-associated phospholipase A2 (Lp-PLA2) and E-selectin in patients with symptomatic and asymptomatic carotid stenosis.

\section{Materials and methods}

The study included patients, who were hospitalized in the period from November'2018 to January 2020 at the City Clinical Hospital No. 7 of the Kharkiv City Council, Ukraine.

The 106 patients with atherosclerotic carotid stenosis (74 men and 32 women, aged from 31 to 74 years, mean $62.6 \pm 0.9$ ) were involved in the study. The criterion for involving were atherosclerotic carotid stenosis $>50 \%$, absence history of atrial fibrillation, intracranial haemorrhage, inflammatory diseases, cancer. The symptomatic groups included 35 patients (group 1) had presented with acute non-lacunar ischaemic stroke in ipsilateral internal carotid artery (ICA), NIHSS 3-9 and 41 patients (group 2) who suffered from ischaemic stroke and underwent carotid endarterectomy (CEA) on ipsilateral side with the mean follow-up period 35 months. When determining the indications for surgical intervention guided by generally accepted recommendations. Asymptomatic group (group 3) included 30 patients who had acute ischemic event in clinical history. Neuroimaging (CT or MRI) was performed in all patients. The control group consisted of 31-56 age- and sex-matched 20 healthy subjects who visited our hospital for a routine physical check-up and without a history of cardiac disease, hypertension or diabetes and having normal findings on physical examination, Doppler ultrasound and echocardiography.

Informed consent was obtained from all participants and the study was approved by the Bioethics Committee of Kharkiv Medical Academy of Postgraduate Education (report No. 9 of $21^{\text {th }}$ November 2018) in accordance with the Code of Ethic of the World Medical Association (Helsinki Declaration).

In all patients' traditional risk factors as arterial hypertension, smoking, overweight, diabetes mellitus, myocardial infarction history and hypercholesterinemia was assessed.

All patients underwent duplex sonography. Carotid atherosclerotic examinations were performed with a high-resolution (10.0 MHz) color Doppler ultrasound by scanner «MINDRAY DC-40». The degree of ICA stenosis was determined by the European Carotid Surgery Trial (ECST) method [6].

The level of serum Lp-PLA2 was determined using a commercially available enzyme-linked immunosorbent assay kit (ELISA) Elabscience, USA. E-selectin was measured using commercially available (ELISA) kit (Elabscience, USA).

Descriptive statistics of data are presented as $\mathrm{M} \pm \mathrm{m}$ or Me [LQ; UQ] for continuous variables (where $\mathrm{M}$ - mean value, $\mathrm{m}$ - standard error of mean, Me - median, LQ and UQ - lower and upper quartiles). Counts and proportions (\%) are reported for qualitative variables. To test significance of differences of normally distributed quantitative variables between several groups of patients Fisher's ANOVA was applied (the obtained value of test statistic is denoted by F further in the paper). In cases when within-group distributions deviate from normality non-parametric Kruskal-Wallis ANOVA was used (the obtained values of test statistic is denoted by KW $\mathrm{H}$ ) for the same purpose. To compare numerical values in two groups of patients Mann-Whitney U-test was applied (the values of test statistic is denoted by MW U in the paper). To test significance of 
differences between groups for categorical variables Pearson's $\chi^{2}$ test for $r \times c$ contingency tables was applied (the values of test statistic is denoted by $\chi^{2}$ in the paper). The correlations between variables were assessed by Spearman correlation coefficient. The significance of Lp-PLA 2 and E-selectin in determining the presence of stroke was assessed by the analysis of ROC-curves (Receiver Operating Characteristics) of logistic regression models. The statistical analysis and inference was performed at $95 \%$ confidence probability, so p-values less than 0.05 were considered statistically significant. STATISTICA 13 software package (TIBCO Software Inc., USA) was used for data analysis.

\section{Results}

Baseline and clinical characteristics of patients with carotid stenosis are shown in Table $\mathbf{1}$. All patients had $\geq 1$ atherosclerotic risk factors. It showed a trend toward a higher prevalence of current smoking, high total cholesterol level and diabetes mellitus in symptomatic patients groups with acute stroke and history of stroke and CEA.

Table 1

Baseline and clinical characteristics patients with carotid artery stenosis

\begin{tabular}{|c|c|c|c|c|}
\hline Variable & $\begin{array}{c}\text { Total } \\
n=106\end{array}$ & $\begin{array}{c}\text { Group } 1 \\
n=35\end{array}$ & $\begin{array}{c}\text { Group } 2 \\
n=41\end{array}$ & $\begin{array}{c}\text { Group } 3 \\
n=\mathbf{3 0}\end{array}$ \\
\hline Age, year & $62.6 \pm 0.9$ & $61.6 \pm 1.5$ & $59.7 \pm 1.3$ & $67.7 \pm 1.8^{*}$ \\
\hline Gender, male/female & $74(69.8 \%) / 32(30.2 \%)$ & $29(82.9 \%) / 6(17.1 \%)$ & $33(80.5 \%) / 8(19.5 \%)$ & $12(40.0 \%) / 18(60.0 \%) *$ \\
\hline Arterial Hypertension & $89 / 84.0 \%$ & $31 / 89.0 \%$ & $30 / 73.2 \%$ & $28 / 93.3 \%$ \\
\hline Current smokers & $64 / 60.4 \%$ & $30 / 85.7 \%$ & $22 / 53.6 \%$ & $12 / 40.0 \% *$ \\
\hline $\begin{array}{l}\text { Total cholesterol } \\
\quad \geq 5 \mathrm{mmol} / 1\end{array}$ & $63 / 67.7 \%$ & $23 / 65.7 \%$ & $34 / 82.9 \%$ & $17 / 56.6 \%$ \\
\hline $\begin{array}{l}\text { Low density cholesterol } \\
\qquad \geq 2 \mathrm{mmol} / 1\end{array}$ & $87 / 93.5 \%$ & $33 / 94.3 \%$ & $35 / 85.4 \%$ & $30 / 100.0 \%$ \\
\hline Body mass index $\geq 25$ & $70 / 66.0 \%$ & $25 / 71.4 \%$ & $25 / 61.0 \%$ & $20 / 66.7 \%$ \\
\hline Diabetes mellitus & $7 / 6.6 \%$ & $4 / 11.4 \%$ & $2 / 4.9 \%$ & $1 / 3.3 \%$ \\
\hline $\begin{array}{l}\text { Myocardial infarction } \\
\text { history }\end{array}$ & $10 / 9.4 \%$ & $3 / 8.6 \%$ & $3 / 7.3 \%$ & $4 / 13.3 \%$ \\
\hline \multicolumn{5}{|c|}{ Degree of ipsilateral carotid artery stenosis } \\
\hline $50-69 \%$ & $49 / 46.2 \%$ & $20 / 57.1 \%$ & $6 / 14.6 \%$ & $23 / 76.7 \% *$ \\
\hline $70-99 \%$ & $41 / 38.7 \%$ & $8 / 22.9 \%$ & $26 / 63.4 \%$ & $7 / 23.3 \% *$ \\
\hline occlusion & $15 / 14.2 \%$ & $7 / 20.0 \%$ & $8 / 29.5 \%$ & $0 / 0.0 \%$ \\
\hline
\end{tabular}

Note: $*_{-}^{*}<0.05$ comparison between patients with symptomatic and asymptomatic carotid stenosis

The level of Lp-PLA 2 in patients was on average $46.02[29.51 ; 79.71] \mathrm{ng} / \mathrm{ml}$, which was significantly higher $\left(\mathrm{MW} \mathrm{U}=10, p=1.023136 \times 10^{-11}<0.05\right)$ than in the control group, in which Lp-PLA 2 was observed at the level of $9.92[4.58 ; 12.48] \mathrm{ng} / \mathrm{ml}$. Lp-PLA 2 concentration was significantly higher in groups of symptomatic patients who underwent CEA. Lp-PLA 2 did not differ in men and women (MW U $=495.0, p=0.06387>0.05)$ (Table 2).

The level of E-selectin in the study patients was significantly higher than in the control group ( $\mathrm{MW} \mathrm{U}=113.0, p=3.3011 \times 10^{-9}<0.05$ ), however, the differences between the three study groups were insignificant $(\mathrm{KW} \mathrm{H}(2.80)=0.7979, p=0.6710>0.05)$ (Table 2).

The correlation of Lp-PLA 2 with E-selectin, assessed for total patients, was significant $(p=0.00085)$. In symptomatic group in patients after CEA, the correlation of E-selectin with LP-PLA 2 was also statistically significant $(p=0.01796)$. However in the group of asymptomatic stenosis this correlation was absent $(p=0.13977)$.

Three models of logistic regression to predict the presence of a stroke were developed and studied: 1) a one-dimensional model of the dependence of the probability of stroke on the values of Lp-PLA 2; 2) one-dimensional model of the dependence of the probability of stroke on the values of E-selectin; 3) a multidimensional model of the dependence of the probability of stroke on a linear 
combination of Lp-PLA 2 and E-selectin. The significance of the studied indicators for predicting the development of stroke was assessed by the value of the area under the ROC-curve, as well as the overall accuracy, specificity and sensitivity of the obtained model were taken into consideration.

Table 2

Serum level Lp-PLA 2 and E-selectin in patients with atherosclerotic carotid stenosis with different clinical manifestation

\begin{tabular}{ccc}
\hline Group of patients & $\begin{array}{c}\text { Lp-PLA2 }(\mathbf{n g} / \mathbf{m l}) \\
\mathbf{M} \pm \mathbf{m}\end{array}$ & $\begin{array}{c}\text { E-selectin }(\mathbf{p g} / \mathbf{m l}) \\
\mathbf{M} \pm \mathbf{m}\end{array}$ \\
\hline Control $n=20$ & $9.296 \pm 0.935$ & $3.101 \pm 0.503$ \\
Total $n=106$ & $55.664 \pm 3.537^{*}$ & $7.653 \pm 0.246^{*}$ \\
Group $1 n=35$ & $46.412 \pm 6.421^{*}$ & $8.070 \pm 0.430^{*}$ \\
Group $2 n=41$ & $67.645 \pm 5.742^{*} * *$ & $7.497 \pm 0.408^{*}$ \\
Group $3 n=30$ & $50.539 \pm 5.560^{*}$ & $7.423 \pm 0.446^{*}$
\end{tabular}

Note: $*-p<0.05$ comparison between patient's group and control; $* *-p<0.05$ comparison between group 1 and group 2

To predict the rate of occurrence of the stroke in fallowness from the level of Lp-PLA 2, the following model is adopted:

$$
\begin{gathered}
P_{\text {stroke }}=\frac{1}{1+e^{y(\text { Lp_PLA } 2)}} ; \\
y(\text { Lp_PLA } 2)=0.987-0.272 \cdot \text { LP_PLA } 2 .
\end{gathered}
$$

The statistical significance of the model coefficients assessed by Wald's criterion allows us to conclude that the increase in the level of Lp-PLA 2 has a significant impact on the increase in the probability of stroke ( $p=0.00039)$. The overall prediction accuracy is $67 \%$, but the model has a fairly balanced value of specificity and sensitivity, which is expressed by $\mathrm{AUC}=0.7535$, which indicates the average quality of the model (Fig. 1).

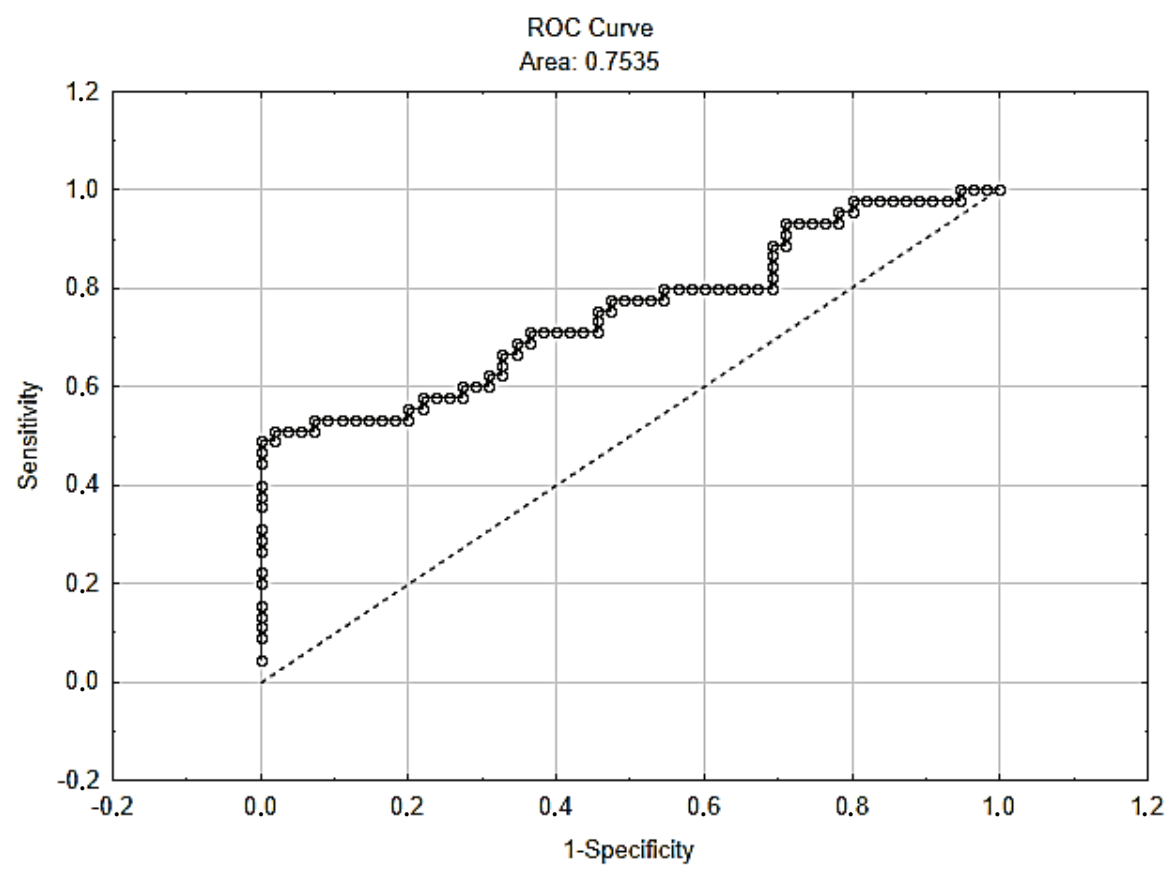

Fig. 1. ROC-curve of the logistic regression model for predicting the presence of stroke based on the level of Lp-PLA 2 
To predict the risk of a stroke in the presence of an E-selectin, the following model was adopted:

$$
\begin{gathered}
P_{\text {stroke }}=\frac{1}{1+e^{y\left(E_{\text {selectin }}\right)}} ; \\
y\left(E_{\text {selectin }}\right)=2.0595-0.3383 \cdot E_{\text {selectin }} .
\end{gathered}
$$

The statistical significance of the coefficients of the model suggests that increasing the level of E-selectin has a significant impact on increasing the risk of stroke $(p=0.00029)$.

Overall prediction accuracy of $70 \%$, but the prediction of stroke probability based on E-selectin has less balanced values of specificity and sensitivity than a similar model based on the level of LP-FLA 2, which is expressed by AUC $=0.7002$ (Fig. 2). This value of the area under the ROC curve indicates the mediocre quality of the model for predicting the occurrence of stroke on the basis of E-selectin.

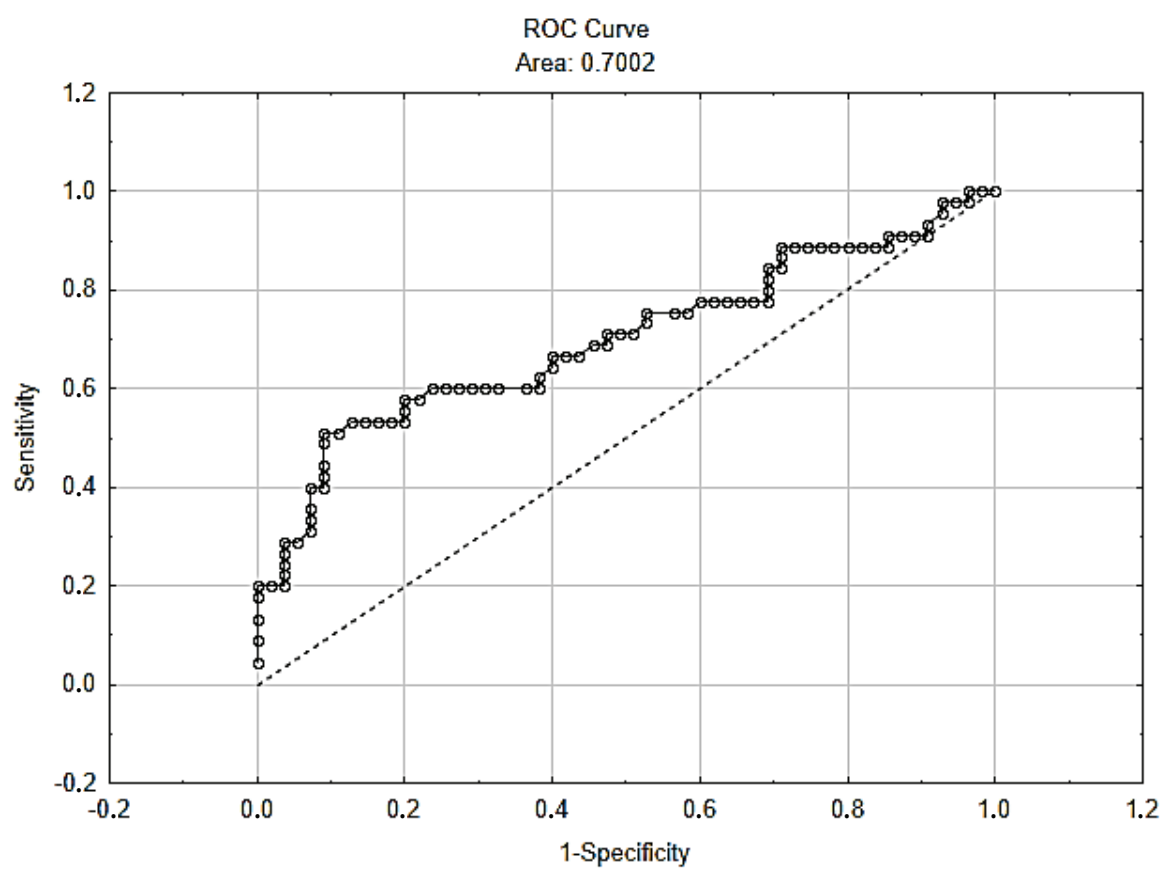

Fig. 2. ROC-curve of the logistic regression model for predicting the presence of stroke based on the level of E-selectin

We have also studied a model of logistic regression, which is approximate to the visibility of a stroke on the basis of a linear combination of E-selectin and Lp-PLA 2.

$$
\begin{gathered}
P_{\text {stroke }}=\frac{1}{1+e^{y\left(E_{\text {selectin }}, \text { Lp_PLA }\right)}} ; \\
y\left(E_{\text {selectin }}, \mathrm{LP} \_ \text {PLAP } 2\right)=2.1392-0.2364 \cdot E_{\text {selectin }}-0.0172 \cdot \mathrm{Lp} \_ \text {PLA } 2 .
\end{gathered}
$$

The model, which takes into joint both indicators, also confirms the statistical significance of the influence of the studied factors on the probability of stroke. Having a slightly higher Nagelkerke coefficient $R^{2}=0.2604$ than one-dimensional models, this model is characterized by $70 \%$ of the overall accuracy of stroke detection and $\mathrm{AUC}=0.7482$, which indicates the average quality of the model (Fig. 3).

Thus, studies conducted show that the levels of Lp-PLA 2 and E-selectin have a significant impact on the development of stroke and can be used to predict it. The combination of Lp-PLA 2 and E-selectin allows to obtain significantly higher characteristics of the accuracy of stroke prediction. 


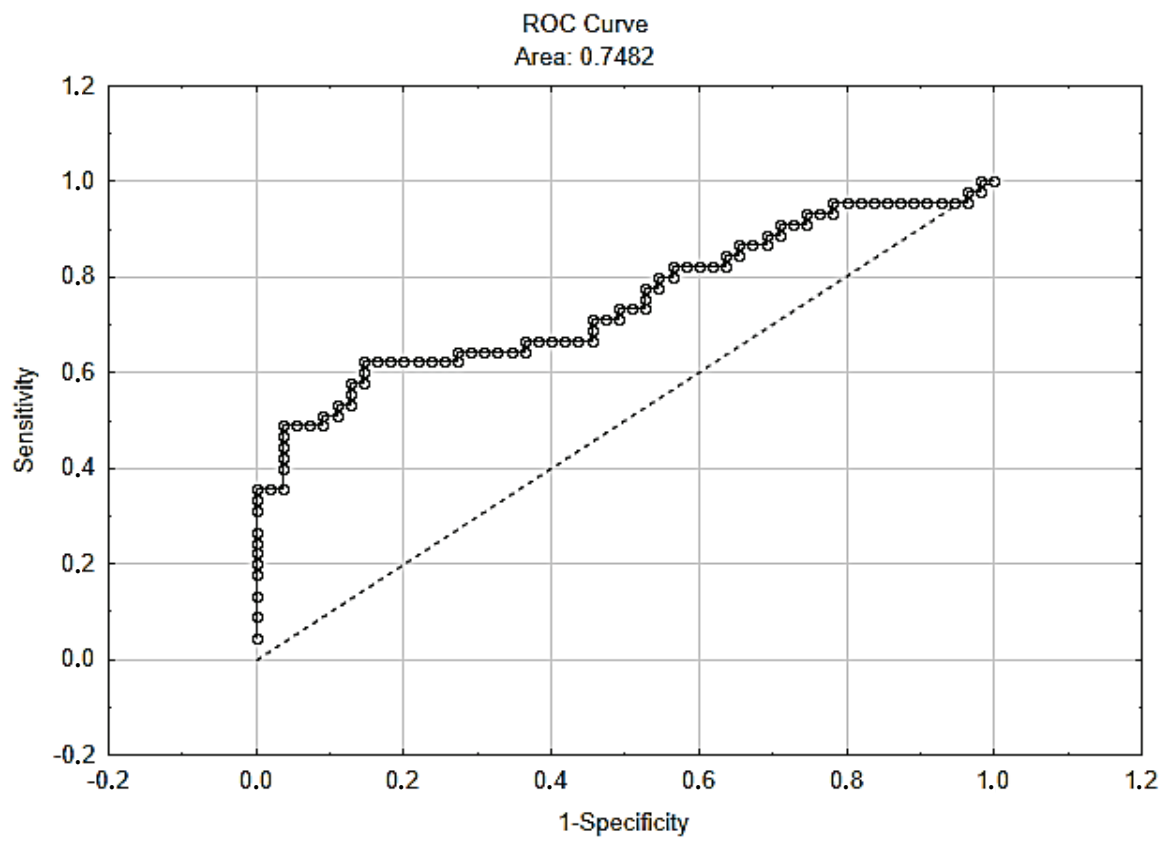

Fig. 3. ROC-curve of the logistic regression model for predicting the presence of stroke based on a linear combination of levels of E-selectin and Lp-PLA 2

\section{Discussion}

The biological mechanisms involving plasma Lp-PLA 2 in the pathogenesis of the atherosclerosis are not well-characterized, however there are sufficiency evidence that Lp-PLA2 involved in the process of destabilizing atherosclerotic plaques by increasing inflammatory activity [7]. Furthermore, atherosclerosis is now recognized as manifestations of vascular inflammation and atherosclerotic plaque destabilization and rupture and successive clinical complications depend on several inflammatory molecules involved in the atherosclerosis pathogenesis [8]. Lp-PLA 2, originally named platelet-activating factor acetylhydrolase (PAF-AH), is an enzyme involved in lipoprotein metabolism and inflammatory pathways. In human, $80 \%$ of Lp-PLA 2 circulates bound to LDL-cholesterol, 10-15\% circulates with HDL-cholesterol, and the remaining 5-10\% circulates with VLDL-cholesterol. Lp-PLA 2 enzymatic activity results in generation of lysophosphotidylcholine (lysoPC) and oxidized non-esterified fatty acids, two pro-inflammatory mediators [9]. Several studies have shown that Lp-PLA2 may be a biological marker that is associated with lipoprotein metabolism, vascular inflammation and destabilization of atherosclerotic plaque, leading to the development of cardiovascular diseases [10]. High Lp-PLA levels have been associated with a high risk of cardiovascular events in healthy older adults [11]. An increased level of circulating Lp-PLA 2 was found in patients with high-grade carotid stenosis and unstable plaques who underwent carotid endarterectomy [12]. With the development of atherosclerosis, adhesion of monocytes on the surface of the endothelium is activated by adhesion molecules E-selectin and ICAM-1 [13]. In previous study was shown that in atherosclerotic plaque in patients after CEA to observe endothelian expression of molecules adhesion VCAM and E-selectin that reflect the macrophage burden within plaque lesion and inflammatory activity of atherosclerosis [14]. Inflammatory factors such as adhesion molecules (ICAM-1 and VCAM-1), CD40 ligands, C-reactive protein and myeloperoxidase (MPO) participate in induction of atherosclerotic disease. The lysoPC stimulates macrophage proliferation, up-regulates cytokines and CD40 ligands, and increases the expression of vascular adhesion molecules, implying a complex interaction between Lp-PLA 2 and other inflammatory mediators [15]. In this study most high level of Lp-PLA 2 was observed in symptomatic patients who underwent CEA after ipsilateral stroke. Patients of this group was younger, prevalent male with severe carotid stenosis. Closer correlations between markers of atherogenesis in this group may indicate on more aggressive course of atherosclerosis 
in these patients. In our study Lp-PLA 2 has been implicated in inflammation and considered as an inflammatory marker that increased in relationship with activation adhesion molecules E-selectin. Therefore it is concluded that there is a significant synergistic relationship between E-selectin endothelial expression and Lp-PLA 2 in patients with severe degree stenosis and high risk of acute cerebrovascular events.

CEA is a proven secondary prophylaxis method for severe carotid stenosis [16, 17], but restenosis is often observed after surgery, which increases the risk of stroke [18]. Asymptomatic carotid artery stenosis is a well-recognized risk factor for ischemic stroke and its prevalence increases with severity of degree of stenosis [19]. With stroke risk associated also ultrasound plaque features, but best treatment for asymptomatic carotid artery stenosis and prophylactic revascularization still controversial [20]. To identify subgroup of patients at higher risk for ipsilateral stroke is important aim. Individual's tendency to develop inflammation-dependent vascular disease might be related to a susceptibility profile based on functional interactions among a number of different markers. In our study clarified that the levels of Lp-PLA 2 and E-selectin have a significant impact on the development of stroke and can be used to predict it.

Study limitation. First, this study had a relative small sample. Second, estimation of stroke occurrence needs long duration of follow-up.

Prospects for further research. These finding warrant further investigators for estimate prognostic role atherosclerotic markers for risk of stroke occurrence. Elevation of atherosclerotic markers in patients with high risk of stroke may be a potential therapeutic target for prevention strategy.

\section{Conclusion}

The present study identified elevated total plasma Lp-PLA 2 level in patients with carotid artery stenosis comparable with control group $(p<0.05)$. Lp-PLA 2 concentration was significantly higher in group of symptomatic patients who underwent CEA $(p<0.05)$.

The level of E-selectin in study patients was significantly higher than in control group $(p<0.05)$, but the differences between groups are absence.

We demonstrated that the Lp-PLA 2 correlated with serum level of E-selectin $(p<0.05)$. The logistic regression model showed that combination of Lp-PLA 2 and E-selectin allows to predicting the stroke development with $70 \%$ of the accuracy in patients with carotid artery stenosis.

\section{Conflict of interests}

The authors declare that they have no conflicts of interest.

\section{Financing}

The study was performed without financial support.

\section{Acknowledgments}

We express our gratitude to the patients who took part in the study, as well as the specialists who contribute to the investigation.

\section{References}

[1] Messas, E., Goudot, G., Halliday, A., Sitruk, J., Mirault, T., Khider, L. et. al. (2020). Management of carotid stenosis for primary and secondary prevention of stroke: state-of-the-art 2020: a critical review. European Heart Journal Supplements, 22 (Supplement_M), M35-M42. doi: http://doi.org/10.1093/eurheartj/suaa162

[2] Den Hartog, A. G., Achterberg, S., Moll, F. L., Kappelle, L. J., Visseren, F. L. J., van der Graaf, Y. et. al. (2013). Asymptomatic Carotid Artery Stenosis and the Risk of Ischemic Stroke According to Subtype in Patients With Clinical Manifest Arterial Disease. Stroke, 44 (4), 1002-1007. doi: http://doi.org/10.1161/strokeaha.111.669267

[3] Libby, P. (2012). Inflammation in Atherosclerosis. Arteriosclerosis, Thrombosis, and Vascular Biology, 32 (9), $2045-2051$. doi: http://doi.org/10.1161/atvbaha.108.179705

[4] Mayer, F. J., Binder, C. J., Wagner, O. F., Schillinger, M., Minar, E., Mlekusch, W. et. al. (2016). Combined Effects of Inflammatory Status and Carotid Atherosclerosis. Stroke, 47 (12), 2952-2958. doi: http://doi.org/10.1161/strokeaha.116.013647 
[5] Martinez, E., Martorell, J., Riambau, V. (2020). Review of serum biomarkers in carotid atherosclerosis. Journal of Vascular Surgery, 71 (1), 329-341. doi: http://doi.org/10.1016/j.jvs.2019.04.488

[6] Rothwell, P. M., Gutnikov, S. A., Warlow, C. P. (2003). Reanalysis of the Final Results of the European Carotid Surgery Trial. Stroke, 34 (2), 514-523. doi: http://doi.org/10.1161/01.str.0000054671.71777.c7

[7] Toth, P. P., McCullough, P. A., Wegner, M. S., Colley, K. J. (2010). Lipoprotein-associated phospholipase A2: role in atherosclerosis and utility as a cardiovascular biomarker. Expert Review of Cardiovascular Therapy, 8 (3), $425-438$. doi: http://doi.org/10.1586/erc.10.18

[8] Ammirati, E., Moroni, F., Norata, G. D., Magnoni, M., Camici, P. G. (2015). Markers of Inflammation Associated with Plaque Progression and Instability in Patients with Carotid Atherosclerosis. Mediators of Inflammation, 2015, 1-15. doi: http://doi.org/10.1155/2015/718329

[9] Marnane, M., Prendeville, S., McDonnell, C., Noone, I., Barry, M., Crowe, M. et. al. (2014). Plaque Inflammation and Unstable Morphology Are Associated With Early Stroke Recurrence in Symptomatic Carotid Stenosis. Stroke, 45 (3), $801-806$. doi: http://doi.org/10.1161/strokeaha.113.003657

[10] Wang, C., Fang, X., Hua, Y., Liu, Y., Zhang, Z., Gu, X. et. al. (2017). Lipoprotein-Associated Phospholipase A2 and Risk of Carotid Atherosclerosis and Cardiovascular Events in Community-Based Older Adults in China. Angiology, 69 (1), $49-58$. doi: http://doi.org/10.1177/0003319717704554

[11] Kleber, M. E., Siekmeier, R., Delgado, G., Grammer, T. B., Winkelmann, B. R., Scharnagl, H. et. al. (2014). C-Reactive Protein and Lipoprotein-Associated Phospholipase A2 in Smokers and Nonsmokers of the Ludwigshafen Risk and Cardiovascular Health Study. Advances in Experimental Medicine and Biology, 832, 15-23. doi: http://doi.org/10.1007/5584_2014_6

[12] Sarlon-Bartoli, G., Boudes, A., Buffat, C., Bartoli, M. A., Piercecchi-Marti, M. D., Sarlon, E. (2012). Circulating Lipoprotein-associated Phospholipase A2 in High-grade Carotid Stenosis: A New Biomarker for Predicting Unstable Plaque. European Journal of Vascular and Endovascular Surgery, 43 (2), 154-159. doi: http://doi.org/10.1016/j.ejvs.2011.10.009

[13] Wijeratne, T., Menon, R., Sales, C., Karimi, L., Crewther, S. (2020). Carotid artery stenosis and inflammatory biomarkers: the role of inflammation-induced immunological responses affecting the vascular systems. Annals of Translational Medicine, 8 (19), 1276-1276. doi: http://doi.org/10.21037/atm-20-4388

[14] Chan, J. M. S., Monaco, C., Wylezinska-Arridge, M., Tremoleda, J. L., Gibbs, R. G. J. (2014). Imaging of the Vulnerable Carotid Plaque: Biological Targeting of Inflammation in Atherosclerosis using Iron Oxide Particles and MRI. European Journal of Vascular and Endovascular Surgery, 47 (5), 462-469. doi: http://doi.org/10.1016/j.ejvs.2014.01.017

[15] Puz, P., Lasek-Bal, A., Ziaja, D., Kazibutowska, Z., Ziaja, K. (2013). Inflammatory markers in patients with internal carotid artery stenosis. Archives of Medical Science, 2, 254-260. doi: http://doi.org/10.5114/aoms.2013.34533

[16] Shah, Z., Masoomi, R., Thapa, R., Wani, M., Chen, J., Dawn, B. et. al. (2017). Optimal Medical Management Reduces Risk of Disease Progression and Ischemic Events in Asymptomatic Carotid Stenosis Patients: A Long-Term Follow-Up Study. Cerebrovascular Diseases, 44 (3-4), 150-159. doi: http://doi.org/10.1159/000477501

[17] Kakkos, S. K., Kakisis, I., Tsolakis, I. A., Geroulakos, G. (2017). Endarterectomy achieves lower stroke and death rates compared with stenting in patients with asymptomatic carotid stenosis. Journal of Vascular Surgery, 66 (2), $607-617$. doi: http://doi.org/10.1016/j.jvs.2017.04.053

[18] Bonati, L. H., Gregson, J., Dobson, J., McCabe, D. J. H., Nederkoorn, P. J., van der Worp, H. B. et. al. (2018). Restenosis and risk of stroke after stenting or endarterectomy for symptomatic carotid stenosis in the International Carotid Stenting Study (ICSS): secondary analysis of a randomised trial. The Lancet Neurology, 17 (7), 587-596. doi: http://doi.org/10.1016/ s1474-4422(18)30195-9

[19] King, A., Shipley, M., Markus, H. (2013). The Effect of Medical Treatments on Stroke Risk in Asymptomatic Carotid Stenosis. Stroke, 44 (2), 542-546. doi: http://doi.org/10.1161/strokeaha.112.673608

[20] Goudot, G., Khider, L., Pedreira, O., Poree, J., Julia, P., Alsac, J.-M. et. al. (2020). Innovative Multiparametric Characterization of Carotid Plaque Vulnerability by Ultrasound. Frontiers in Physiology, 11. doi: http://doi.org/10.3389/fphys.2020.00157

How to cite: Dubenko, O., Litovchenko, T., Anysienkova, V., Nessonova, M., Kovalenko, L. (2021). The prognostic value of the serum inflammatory biomarkers in patients with carotid atherosclerosis. EUREKA: Health Sciences, 4, 26-33. doi: http://doi.org/ 10.21303/2504-5679.2021.001969 